\title{
On the Cauchy Functional Inequality in Banach Modules
}

\author{
Choonkil Park \\ Department of Mathematics, Hanyang University, Seoul 133791, South Korea \\ Correspondence should be addressed to Choonkil Park, baak@hanyang.ac.kr \\ Received 17 January 2008; Revised 21 March 2008; Accepted 16 April 2008 \\ Recommended by Ram Mohapatra \\ We investigate the following functional inequality: $\|f(x)+f(y)+f(z)\| \leq\|f(x+y+z)\|$ in Banach \\ modules over a $C^{*}$-algebra, and prove the generalized Hyers-Ulam stability of linear mappings in \\ Banach modules over a $C^{*}$-algebra. \\ Copyright (C) 2008 Choonkil Park. This is an open access article distributed under the Creative \\ Commons Attribution License, which permits unrestricted use, distribution, and reproduction in \\ any medium, provided the original work is properly cited.
}

\section{Introduction and preliminaries}

The stability problem of functional equations originated from a question of Ulam [1] concerning the stability of group homomorphisms. Hyers [2] gave a first affirmative partial answer to the question of Ulam for Banach spaces. Let $X$ and $Y$ be Banach spaces. Hyers' theorem was generalized by Aoki [3] for additive mappings and by Th. M. Rassias [4] for linear mappings by considering an unbounded Cauchy difference. A generalization of the Th. M. Rassias theorem was obtained by Găvruţa [5] by replacing the unbounded Cauchy difference by a general control function in the spirit of Th. M. Rassias' approach. The result of Găvruţa [5] is a special case of a more general theorem, which was obtained by Forti [6].

Th. M. Rassias [7] during the 27th international symposium on functional equations asked the question whether such a theorem can also be proved for $p \geq 1$. Gajda [8], following the same approach as in Th. M. Rassias [4], gave an affirmative solution to this question for $p>1$. It was shown by Gajda [8], as well as by Th. M. Rassias and Šemrl [9] that one cannot prove a Th. M. Rassias'-type theorem when $p=1$.

J. M. Rassias [10] followed the innovative approach of Th. M. Rassias' theorem in which he replaced the factor $\|x\|^{p}+\|y\|^{p}$ by $\|x\|^{p} \cdot\|y\|^{q}$ for $p, q \in \mathbb{R}$ with $p+q \neq 1$.

During the last two decades, a number of papers and research monographs have been published on various generalizations and applications of the generalized Hyers-Ulam stability to a number of functional equations and mappings (see [11-21]). 
Journal of Inequalities and Applications

Gilányi [22] showed that if $f$ satisfies the functional inequality

$$
\|2 f(x)+2 f(y)-f(x-y)\| \leq\|f(x+y)\|,
$$

then $f$ satisfies the Jordan-von Neumann functional equation

$$
2 f(x)+2 f(y)=f(x+y)+f(x-y) .
$$

See also [23]. Fechner [24] and Gilányi [25] proved the generalized Hyers-Ulam stability of the functional inequality (1.1). Park et al. [19] investigated the functional inequality

$$
\|f(x)+f(y)+f(z)\| \leq\|f(x+y+z)\|
$$

in Banach spaces, and proved the generalized Hyers-Ulam stability of the functional inequality (1.3) in Banach spaces.

Throughout this paper, let $A$ be a unital $C^{*}$-algebra with unitary group $U(A)$ and unit $e$. Assume that $X$ is a Banach $A$-module with norm $\|\cdot\|_{X}$ and that $Y$ is a Banach $A$-module with norm $\|\cdot\|_{Y}$.

In this paper, we investigate an $A$-linear mapping associated with the functional inequality (1.3) and prove the generalized Hyers-Ulam stability of $A$-linear mappings in Banach $A$-modules associated with the functional inequality (1.3).

The computations in the proofs of the main theorems are special cases of the general results obtained by Forti [26].

\section{Functional inequalities in Banach modules over a $C^{*}$-algebra}

Lemma 2.1. Let $f: X \rightarrow Y$ be a mapping such that

$$
\|f(x)+f(y)+u f(z)\|_{Y} \leq\|f(x+y+u z)\|_{Y}
$$

for all $x, y, z \in X$ and all $u \in U(A)$. Then $f$ is A-linear.

Proof. Letting $x=y=z=0$ and $u=e \in U(A)$ in (2.1), we get

$$
\|3 f(0)\|_{Y} \leq\|f(0)\|_{Y} \text {. }
$$

So, $f(0)=0$.

Letting $z=0$ and $y=-x$ in (2.1), we get

$$
\|f(x)+f(-x)\|_{Y} \leq\|f(0)\|_{Y}=0
$$

for all $x \in X$. Hence $f(-x)=-f(x)$ for all $x \in X$.

Letting $z=-x-y$ and $u=e \in U(A)$ in (2.1), we get

$$
\|f(x)+f(y)-f(x+y)\|_{Y}=\|f(x)+f(y)+f(-x-y)\|_{Y} \leq\|f(0)\|_{Y}=0
$$

for all $x, y \in X$. Thus,

$$
f(x+y)=f(x)+f(y)
$$

for all $x, y \in X$. 
Letting $x=-u z$ and $y=0$ in (2.1), we get

$$
\|-f(u z)+u f(z)\|_{Y}=\|f(-u z)+u f(z)\|_{Y} \leq\|f(0)\|_{Y}=0
$$

for all $z \in X$ and all $u \in U(A)$. Thus,

$$
f(u z)=u f(z)
$$

for all $u \in U(A)$ and all $z \in X$.

Now let $a \in A(a \neq 0)$ and $M$ an integer greater than $4|a|$. Then $|a / M|<1 / 4<1-2 / 3=$ $1 / 3$. By [27, Theorem 1], there exist three elements $u_{1}, u_{2}, u_{3} \in U(A)$ such that $3(a / M)=$ $u_{1}+u_{2}+u_{3}$. So by $(2.7)$

$$
\begin{aligned}
f(a x) & =f\left(\frac{M}{3} \cdot 3 \frac{a}{M} x\right)=M \cdot f\left(\frac{1}{3} \cdot 3 \frac{a}{M} x\right)=\frac{M}{3} f\left(3 \frac{a}{M} x\right)=\frac{M}{3} f\left(u_{1} x+u_{2} x+u_{3} x\right) \\
& =\frac{M}{3}\left(f\left(u_{1} x\right)+f\left(u_{2} x\right)+f\left(u_{3} x\right)\right)=\frac{M}{3}\left(u_{1}+u_{2}+u_{3}\right) f(x)=\frac{M}{3} \cdot 3 \frac{a}{M} f(x)=a f(x)
\end{aligned}
$$

for all $x \in X$. So, $f: X \rightarrow Y$ is $A$-linear, as desired.

Now, we prove the generalized Hyers-Ulam stability of $A$-linear mappings in Banach A-modules.

Theorem 2.2. Let $r>1$ and $\theta$ be nonnegative real numbers, and let $f: X \rightarrow Y$ be an odd mapping such that

$$
\|f(x)+f(y)+u f(z)\|_{Y} \leq\|f(x+y+u z)\|_{Y}+\theta\left(\|x\|_{X}^{r}+\|y\|_{X}^{r}+\|z\|_{X}^{r}\right)
$$

for all $x, y, z \in X$ and all $u \in U(A)$. Then, there exists a unique A-linear mapping $L: X \rightarrow Y$ such that

$$
\|f(x)-L(x)\|_{Y} \leq \frac{2^{r}+2}{2^{r}-2} \theta\|x\|_{X}^{r}
$$

for all $x \in X$.

Proof. Since $f$ is an odd mapping, $f(-x)=-f(x)$ for all $x \in X$.

Letting $u=e \in U(A), y=x$ and $z=-2 x$ in (2.9), we get

$$
\|2 f(x)-f(2 x)\|_{Y}=\|2 f(x)+f(-2 x)\|_{Y} \leq\left(2+2^{r}\right) \theta\|x\|_{X}^{r}
$$

for all $x \in X$. So,

$$
\left\|f(x)-2 f\left(\frac{x}{2}\right)\right\|_{Y} \leq \frac{2+2^{r}}{2^{r}} \theta\|x\|_{X}^{r}
$$


for all $x \in X$. Hence,

$$
\begin{aligned}
\left\|2^{l} f\left(\frac{x}{2^{l}}\right)-2^{m} f\left(\frac{x}{2^{m}}\right)\right\|_{Y} & \leq \sum_{j=l}^{m-1}\left\|2^{j} f\left(\frac{x}{2^{j}}\right)-2^{j+1} f\left(\frac{x}{2^{j+1}}\right)\right\|_{Y} \\
& \leq \frac{2+2^{r}}{2^{r}} \sum_{j=l}^{m-1} \frac{2^{j}}{2^{r j}} \theta\|x\|_{X}^{r}
\end{aligned}
$$

for all nonnegative integers $m$ and $l$ with $m>l$ and all $x \in X$. It follows from (2.13) that the sequence $\left\{2^{n} f\left(x / 2^{n}\right)\right\}$ is Cauchy for all $x \in X$. Since $Y$ is complete, the sequence $\left\{2^{n} f\left(x / 2^{n}\right)\right\}$ converges. So, one can define the mapping $L: X \rightarrow Y$ by

$$
L(x):=\lim _{n \rightarrow \infty} 2^{n} f\left(\frac{x}{2^{n}}\right)
$$

for all $x \in X$. Moreover, letting $l=0$ and passing the limit $m \rightarrow \infty$ in (2.13), we get (2.10).

It follows from (2.9) that

$$
\begin{aligned}
\|L(x)+L(y)+u L(z)\|_{Y} & =\lim _{n \rightarrow \infty} 2^{n}\left\|f\left(\frac{x}{2^{n}}\right)+f\left(\frac{y}{2^{n}}\right)+u f\left(\frac{z}{2^{n}}\right)\right\|_{Y} \\
& \leq \lim _{n \rightarrow \infty} 2^{n}\left\|f\left(\frac{x+y+u z}{2^{n}}\right)\right\|_{Y}+\lim _{n \rightarrow \infty} \frac{2^{n} \theta}{2^{n r}}\left(\|x\|_{X}^{r}+\|y\|_{X}^{r}+\|z\|_{X}^{r}\right) \\
& =\|L(x+y+u z)\|_{Y}
\end{aligned}
$$

for all $x, y, z \in X$ and all $u \in U(A)$. So,

$$
\|L(x)+L(y)+u L(z)\|_{Y} \leq\|L(x+y+u z)\|_{Y}
$$

for all $x, y, z \in X$ and all $u \in U(A)$. By Lemma 2.1, the mapping $L: X \rightarrow Y$ is $A$-linear.

Now, let $T: X \rightarrow Y$ be another $A$-linear mapping satisfying (2.10). Then, we have

$$
\begin{aligned}
\|L(x)-T(x)\|_{Y} & =2^{n}\left\|L\left(\frac{x}{2^{n}}\right)-T\left(\frac{x}{2^{n}}\right)\right\|_{Y} \\
& \leq 2^{n}\left(\left\|L\left(\frac{x}{2^{n}}\right)-f\left(\frac{x}{2^{n}}\right)\right\|_{Y}+\left\|T\left(\frac{x}{2^{n}}\right)-f\left(\frac{x}{2^{n}}\right)\right\|_{Y}\right) \\
& \leq \frac{2\left(2^{r}+2\right) 2^{n}}{\left(2^{r}-2\right) 2^{n r}} \theta\|x\|_{X^{\prime}}^{r}
\end{aligned}
$$

which tends to zero as $n \rightarrow \infty$ for all $x \in X$. So, we can conclude that $L(x)=T(x)$ for all $x \in X$. This proves the uniqueness of $L$. Thus, the mapping $L: X \rightarrow Y$ is a unique $A$-linear mapping satisfying (2.10). 
Theorem 2.3. Let $r<1$ and $\theta$ be positive real numbers, and let $f: X \rightarrow Y$ be an odd mapping satisfying (2.9). Then, there exists a unique A-linear mapping $L: X \rightarrow Y$ such that

$$
\|f(x)-L(x)\|_{Y} \leq \frac{2+2^{r}}{2-2^{r}} \theta\|x\|_{X}^{r}
$$

for all $x \in X$.

Proof. It follows from (2.11) that

$$
\left\|f(x)-\frac{1}{2} f(2 x)\right\|_{Y} \leq \frac{2+2^{r}}{2} \theta\|x\|_{X}^{r}
$$

for all $x \in X$. Hence,

$$
\begin{aligned}
\left\|\frac{1}{2^{l}} f\left(2^{l} x\right)-\frac{1}{2^{m}} f\left(2^{m} x\right)\right\|_{Y} & \leq \sum_{j=l}^{m-1}\left\|\frac{1}{2^{j}} f\left(2^{j} x\right)-\frac{1}{2^{j+1}} f\left(2^{j+1} x\right)\right\|_{Y} \\
& \leq \frac{2+2^{r}}{2} \sum_{j=l}^{m-1} \frac{2^{r j}}{2^{j}} \theta\|x\|_{X}^{r}
\end{aligned}
$$

for all nonnegative integers $m$ and $l$ with $m>l$ and all $x \in X$. It follows from (2.20) that the sequence $\left\{\left(1 / 2^{n}\right) f\left(2^{n} x\right)\right\}$ is Cauchy for all $x \in X$. Since $Y$ is complete, the sequence $\left\{\left(1 / 2^{n}\right) f\left(2^{n} x\right)\right\}$ converges. So, one can define the mapping $L: X \rightarrow Y$ by

$$
L(x):=\lim _{n \rightarrow \infty} \frac{1}{2^{n}} f\left(2^{n} x\right)
$$

for all $x \in X$. Moreover, letting $l=0$ and passing the limit $m \rightarrow \infty$ in (2.20), we get (2.18).

The rest of the proof is similar to the proof of Theorem 2.2.

Theorem 2.4. Let $r>1 / 3$ and $\theta$ be nonnegative real numbers, and let $f: X \rightarrow Y$ be an odd mapping such that

$$
\|f(x)+f(y)+u f(z)\|_{Y} \leq\|f(x+y+u z)\|_{Y}+\theta \cdot\|x\|_{X}^{r} \cdot\|y\|_{X}^{r} \cdot\|z\|_{X}^{r}
$$

for all $x, y, z \in X$ and all $u \in U(A)$. Then, there exists a unique A-linear mapping $L: X \rightarrow Y$ such that

$$
\|f(x)-L(x)\|_{Y} \leq \frac{2^{r} \theta}{8^{r}-2}\|x\|_{X}^{3 r}
$$

for all $x \in X$.

Proof. Since $f$ is an odd mapping, $f(-x)=-f(x)$ for all $x \in X$.

Letting $u=e \in U(A), y=x$ and $z=-2 x$ in (2.22), we get

$$
\|2 f(x)-f(2 x)\|_{Y}=\|2 f(x)+f(-2 x)\|_{Y} \leq 2^{r} \theta\|x\|_{X}^{3 r}
$$

for all $x \in X$. So,

$$
\left\|f(x)-2 f\left(\frac{x}{2}\right)\right\|_{Y} \leq \frac{2^{r}}{8^{r}} \theta\|x\|_{X}^{3 r}
$$


for all $x \in X$. Hence,

$$
\begin{aligned}
\left\|2^{l} f\left(\frac{x}{2^{l}}\right)-2^{m} f\left(\frac{x}{2^{m}}\right)\right\|_{Y} & \leq \sum_{j=l}^{m-1}\left\|2^{j} f\left(\frac{x}{2^{j}}\right)-2^{j+1} f\left(\frac{x}{2^{j+1}}\right)\right\|_{Y} \\
& \leq \frac{2^{r}}{8^{r}} \sum_{j=l}^{m-1} \frac{2^{j}}{8^{r j}} \theta\|x\|_{X}^{3 r}
\end{aligned}
$$

for all nonnegative integers $m$ and $l$ with $m>l$ and all $x \in X$. It follows from (2.26) that the sequence $\left\{2^{n} f\left(x / 2^{n}\right)\right\}$ is Cauchy for all $x \in X$. Since $Y$ is complete, the sequence $\left\{2^{n} f\left(x / 2^{n}\right)\right\}$ converges. So, one can define the mapping $L: X \rightarrow Y$ by

$$
L(x):=\lim _{n \rightarrow \infty} 2^{n} f\left(\frac{x}{2^{n}}\right)
$$

for all $x \in X$. Moreover, letting $l=0$ and passing the limit $m \rightarrow \infty$ in (2.26), we get (2.23).

The rest of the proof is similar to the proof of Theorem 2.2.

Theorem 2.5. Let $r<1 / 3$ and $\theta$ be positive real numbers, and let $f: X \rightarrow Y$ be an odd mapping satisfying (2.22). Then, there exists a unique A-linear mapping $L: X \rightarrow Y$ such that

$$
\|f(x)-L(x)\|_{Y} \leq \frac{2^{r} \theta}{2-8^{r}}\|x\|_{X}^{3 r}
$$

for all $x \in X$.

Proof. It follows from (2.24) that

$$
\left\|f(x)-\frac{1}{2} f(2 x)\right\|_{Y} \leq \frac{2^{r}}{2} \theta\|x\|_{X}^{3 r}
$$

for all $x \in X$. Hence,

$$
\begin{aligned}
\left\|\frac{1}{2^{l}} f\left(2^{l} x\right)-\frac{1}{2^{m}} f\left(2^{m} x\right)\right\|_{Y} & \leq \sum_{j=l}^{m-1}\left\|\frac{1}{2^{j}} f\left(2^{j} x\right)-\frac{1}{2^{j+1}} f\left(2^{j+1} x\right)\right\|_{Y} \\
& \leq \frac{2^{r}}{2} \sum_{j=l}^{m-1} \frac{8^{r j}}{2^{j}} \theta\|x\|_{X}^{3 r}
\end{aligned}
$$

for all nonnegative integers $m$ and $l$ with $m>l$ and all $x \in X$. It follows from (2.30) that the sequence $\left\{\left(1 / 2^{n}\right) f\left(2^{n} x\right)\right\}$ is Cauchy for all $x \in X$. Since $Y$ is complete, the sequence $\left\{\left(1 / 2^{n}\right) f\left(2^{n} x\right)\right\}$ converges. So, one can define the mapping $L: X \rightarrow Y$ by

$$
L(x):=\lim _{n \rightarrow \infty} \frac{1}{2^{n}} f\left(2^{n} x\right)
$$

for all $x \in X$. Moreover, letting $l=0$ and passing the limit $m \rightarrow \infty$ in (2.30), we get (2.28).

The rest of the proof is similar to the proof of Theorem 2.2. 


\section{Acknowledgments}

This work was supported by Korea Research Foundation Grant KRF-2007-313-C00033 and the author would like to thank the referees for a number of valuable suggestions regarding a previous version of this paper.

\section{References}

[1] S. M. Ulam, A Collection of the Mathematical Problems, Interscience Tracts in Pure and Applied Mathematics, no. 8, Interscience, New York, NY, USA, 1960.

[2] D. H. Hyers, "On the stability of the linear functional equation," Proceedings of the National Academy of Sciences of the United States of America, vol. 27, no. 4, pp. 222-224, 1941.

[3] T. Aoki, "On the stability of the linear transformation in Banach spaces," Journal of the Mathematical Society of Japan, vol. 2, pp. 64-66, 1950.

[4] Th. M. Rassias, "On the stability of the linear mapping in Banach spaces," Proceedings of the American Mathematical Society, vol. 72, no. 2, pp. 297-300, 1978.

[5] P. Găvruţa, "A generalization of the Hyers-Ulam-Rassias stability of approximately additive mappings," Journal of Mathematical Analysis and Applications, vol. 184, no. 3, pp. 431-436, 1994.

[6] G. L. Forti, "An existence and stability theorem for a class of functional equations," Stochastica, vol. 4, no. 1 , pp. 23-30, 1980.

[7] Th. M. Rassias, "Problem 16; 2, Report of the 27th International Symposium on Functional Equations," Aequationes Mathematicae, vol. 39, no. 2-3, pp. 292-293, 1990.

[8] Z. Gajda, "On stability of additive mappings," International Journal of Mathematics and Mathematical Sciences, vol. 14, no. 3, pp. 431-434, 1991.

[9] Th. M. Rassias and P. Šemrl, "On the behavior of mappings which do not satisfy Hyers-Ulam stability," Proceedings of the American Mathematical Society, vol. 114, no. 4, pp. 989-993, 1992.

[10] J. M. Rassias, "On approximation of approximately linear mappings by linear mappings," Journal of Functional Analysis, vol. 46, no. 1, pp. 126-130, 1982.

[11] P. Czerwik, Functional Equations and Inequalities in Several Variables, World Scientific, River Edge, NJ, USA, 2002.

[12] D. H. Hyers, G. Isac, and Th. M. Rassias, Stability of Functional Equations in Several Variables, Progress in Nonlinear Differential Equations and Their Applications, 34, Birkhäuser Boston, Boston, Mass, USA, 1998.

[13] K.-W. Jun and Y.-H. Lee, "A generalization of the Hyers-Ulam-Rassias stability of the pexiderized quadratic equations," Journal of Mathematical Analysis and Applications, vol. 297, no. 1, pp. 70-86, 2004.

[14] S.-M. Jung, Hyers-Ulam-Rassias Stability of Functional Equations in Mathematical Analysis, Hadronic Press, Palm Harbor, Fla, USA, 2001.

[15] C. Park, "On the stability of the linear mapping in Banach modules," Journal of Mathematical Analysis and Applications, vol. 275, no. 2, pp. 711-720, 2002.

[16] C. Park, "Lie *-homomorphisms between Lie $C^{*}$-algebras and Lie $*$-derivations on Lie $C^{*}$-algebras," Journal of Mathematical Analysis and Applications, vol. 293, no. 2, pp. 419-434, 2004.

[17] C. Park, "Homomorphisms between Poisson JC*-algebra," Bulletin of the Brazilian Mathematical Society, vol. 36, no. 1, pp. 79-97, 2005.

[18] C. Park, "Fixed points and Hyers-Ulam-Rassias stability of Cauchy-Jensen functional equations in Banach algebras," Fixed Point Theory and Applications, vol. 2007, Article ID 50175, 15 pages, 2007.

[19] C. Park, Y. S. Cho, and M.-H. Han, "Functional inequalities associated with Jordan-von Neumanntype additive functional equations," Journal of Inequalities and Applications, vol. 2007, Article ID 41820, 13 pages, 2007.

[20] C. Park and J. Cui, "Generalized stability of $C^{*}$-ternary quadratic mappings," Abstract and Applied Analysis, vol. 2007, Article ID 23282, 6 pages, 2007.

[21] C. Park and A. Najati, "Homomorphisms and derivations in $C^{*}$-algebras," Abstract and Applied Analysis, vol. 2007, Article ID 80630, 12 pages, 2007.

[22] A. Gilányi, "Eine zur Parallelogrammgleichung äquivalente Ungleichung," Aequationes Mathematicae, vol. 62, no. 3, pp. 303-309, 2001. 
[23] J. Rätz, "On inequalities associated with the Jordan-von Neumann functional equation," Aequationes Mathematicae, vol. 66, no. 1-2, pp. 191-200, 2003.

[24] W. Fechner, "Stability of a functional inequality associated with the Jordan-von Neumann functional equation," Aequationes Mathematicae, vol. 71, no. 1-2, pp. 149-161, 2006.

[25] A. Gilányi, "On a problem by K. Nikodem," Mathematical Inequalities and Applications, vol. 5, no. 4, pp. 707-710, 2002.

[26] G.-L. Forti, "Comments on the core of the direct method for proving Hyers-Ulam stability of functional equations," Journal of Mathematical Analysis and Applications, vol. 295, no. 1, pp. 127-133, 2004.

[27] R. V. Kadison and G. K. Pedersen, "Means and convex combinations of unitary operators," Mathematica Scandinavica, vol. 57, no. 2, pp. 249-266, 1985. 